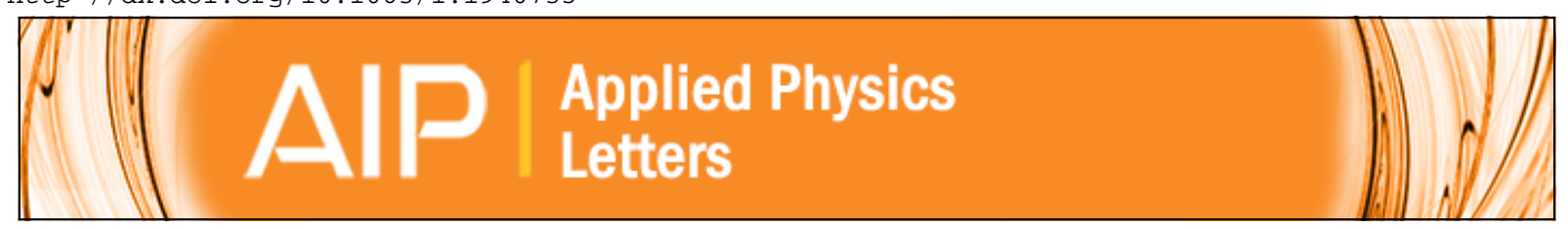

Highly sensitive optical response of optical fiber long period gratings to nanometerthick ionic self-assembled multilayers

Zhiyong Wang, J. R. Heflin, Rogers H. Stolen, and Siddharth Ramachandran

Citation: Applied Physics Letters 86, 223104 (2005); doi: 10.1063/1.1940735

View online: http://dx.doi.org/10.1063/1.1940735

View Table of Contents: http://scitation.aip.org/content/aip/journal/apl/86/22?ver=pdfcov

Published by the AIP Publishing 


\title{
Highly sensitive optical response of optical fiber long period gratings to nanometer-thick ionic self-assembled multilayers
}

\author{
Zhiyong Wang ${ }^{\text {a) }}$ \\ Department of Electrical and Computer Engineering, Virginia Tech, Blacksburg, Virginia 24061 \\ J. R. Heflin ${ }^{\text {b) }}$ \\ Department of Physics, Virginia Tech, Blacksburg, Virginia 24061 \\ Rogers H. Stolen \\ Department of Electrical and Computer Engineering, Virginia Tech, Blacksburg, Virginia 24061 \\ Siddharth Ramachandran ${ }^{\mathrm{c})}$ \\ OFS Laboratories, 19 Schoolhouse Road, Somerset, New Jersey 08873
}

(Received 16 November 2004; accepted 25 April 2005; published online 24 May 2005)

\begin{abstract}
Ionic self-assembled multilayers deposited on long period fiber gratings (LPGs) yield dramatic resonant-wavelength shifts, even with nanometer-thick films. Fine control of the refractive index and the thickness of these films was achieved by altering the relative fraction of the anionic and cationic materials combined with layer-by-layer deposition. We demonstrate the feasibility of this highly controllable deposition technique for fine-tuning grating properties. In addition a variety of biological and chemical sensing agents can easily be incorporated into these films, which makes this an attractive platform for realization of high-performance LPG-based sensors. () 2005 American Institute of Physics. [DOI: 10.1063/1.1940735]
\end{abstract}

Long period fiber gratings (LPGs) couple light between copropagating modes of an optical fiber. They have been used as spectral shapers, ${ }^{1,2}$ and mode converters ${ }^{3}$ in optical fiber communication systems. In addition, LPGs that couple the fundamental mode of a single-mode fiber to one of its cladding modes are ultrasensitive to the refractive index of the material surrounding the fiber, and this high sensitivity and high resolution have led to extensive investigations of LPGs for use as chemical, ${ }^{4}$ biological sensors, and other index-modulating fiber devices. ${ }^{5,6}$

Normally, the index sensitivity is attributed to the index of the bulk medium surrounding the fiber, and features with subwavelength sizes are not expected to modulate the resonance of LPGs. However, Tiefenthaler and Lukosz demonstrated that switching and sensing devices could be achieved by using adsorption or desorption of a monolayer of water molecules on the surface of a short period relief grating coupler fabricated on planar waveguides. ${ }^{7}$ More recently Rees and co-workers ${ }^{8}$ have reported resonant shifts in LPGs with films of subwavelength thickness, using Langmuir-Blodgett (LB) films. The observed optical response was relatively small with maximal shifts of $10 \mathrm{~nm}$ in wavelength with 400 $\mathrm{nm}$ of deposited film. Moreover, LB films are not amenable to practical device construction. This is because the LB technique has demanding requirements of expensive special equipment to precisely control the pressure on the liquid surface and is relatively slow. More significantly, films deposited by the LB technique show poor mechanical and thermal stability because the van Der Waals interaction is the primary binding mechanism.

Ionic self-assembled multilayers (ISAM), on the other hand, are formed by a layer-by-layer deposition technique and exhibit enhanced reliability, stability, and film quality in

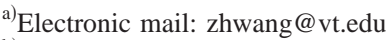

${ }^{b)}$ Electronic mail: rheflin@vt.edu

${ }^{c)}$ Electronic mail: sidr@ieee.org
}

comparison to LB films. ${ }^{9,10}$ The ISAM fabrication method provides a highly controllable means to build precise, nanometer $(\mathrm{nm})$-thick films on the surfaces of fibers (indeed, they can be incorporated on any surface with a minimum charge density, such as metals, glass, or silicon). Moreover, they can be tailored to incorporate a diverse array of particles, such as clay platelets, proteins, virus particles, etc. Thus compared with the LB technique, the ISAM technique shows more flexibility on choices of substrate or template and thin-film overlay materials for devices. Hence, ISAMs on LPGs provide several advantages: (a) ability to fine tune grating resonances in a simple manner, (b) construction of efficient index sensors or index-modulating fiber devices, and (c) application to biosensing.

In this letter, we report ISAM films of nm thicknesses that are deposited on LPGs. Record shifts in the resonant wavelength $(40 \mathrm{~nm})$ were observed for film thicknesses of only $25 \mathrm{~nm}$. Fine control of the refractive index and the thickness of the ISAM film was achieved by altering the relative fraction of the anionic and cationic materials combined with layer-by-layer deposition. We demonstrate the feasibility of this highly controllable deposition technique for fine-tuning grating properties. These demonstrations, combined with the fact that ISAMs can incorporate a variety of chemical and biological sensing elements, provides for a robust platform for building sensors.

The ISAM deposition process involves the immersion of a charged substrate into aqueous solutions of polyanions and polycations in alternating sequence. The nanoscale multilayer thin film is built by consecutive adsorption of polyanions and polycations onto a solid substrate driven by electrostatic forces. The resulting macroscopic properties of the thin film are determined by the properties of individual molecules as well as the dipping sequence of the thin film.

The process is schematically shown in Fig. 1(a). First, a clean, negatively charged substrate is dipped into the polycation solution. Due to the strong coulombic attraction be- 


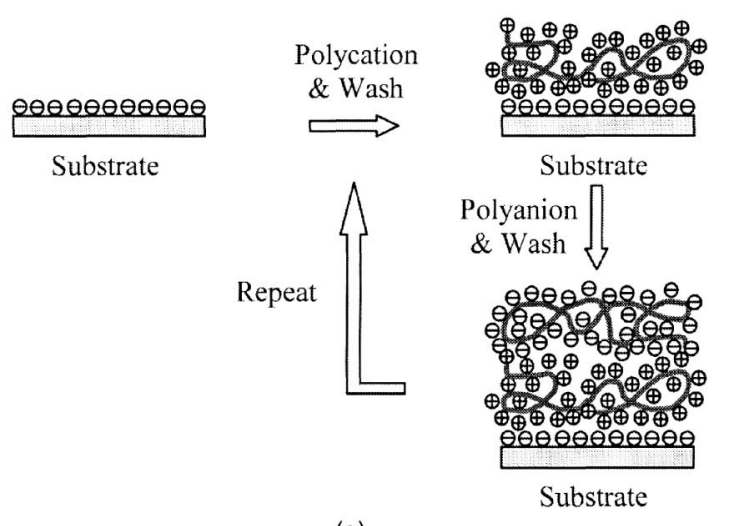

(a)

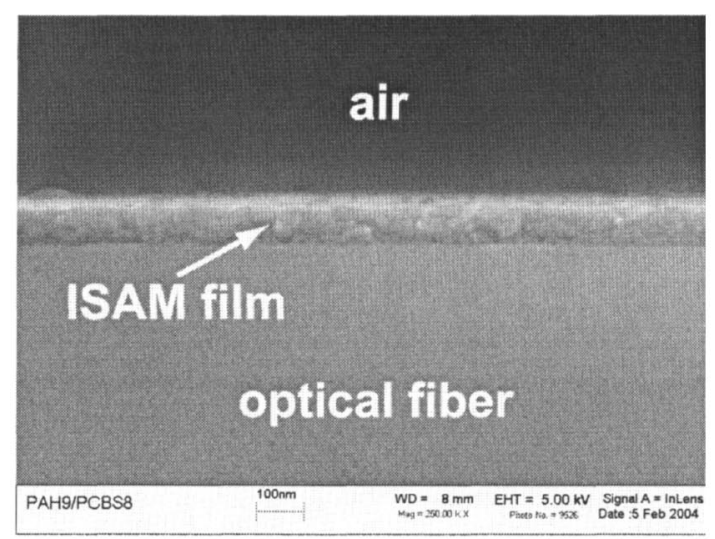

(b)

FIG. 1. (a) Schematic of ISAM film deposition process and illustration of film architecture of the first two layers (one bilayer). (b) Scanning electron microscopy image of ISAM film on an optical fiber.

tween the negatively charged substrate and the positively charged polycation, a layer of polycation molecules is deposited on the substrate. A reversal to positive surface charge results, which limits further polycation adsorption. Then the polycation-coated substrate is removed from the solution and rinsed with de-ionized water to remove excess polycation molecules that are not ionically bound. The substrate is then immersed into the polyanion solution to adsorb a layer of polyanion molecules onto the substrate. Finally, these steps are repeated to obtain a multilayered thin-film with an $(A B)_{n}$ architecture until the desired film thickness is reached. The combination of one layer of polycation and polyanion together is denoted a bilayer. Figure 1(b) is a scanning-electron micrograph of the surface of a fiber coated with an ISAM film, showing characteristic thicknesses achieved by this technique.

In our study, we first investigated the dependence of the properties of ISAM films on the variation of the $p \mathrm{H}$ of the polyanion and polycation solutions by using variable angle spectral ellipsometry. Standard microscope glass slides were used as substrates. Poly(allylamine hydrochloride) (PAH) at $10 \mathrm{mM}$ concentration was used as polycation, and poly\{1-[4(3-carboxy-4-hydroxy-phenylazo) benzenesulfonamido]-1,2ethanediyl, sodium salt\} (PCBS) at $10 \mathrm{mM}$ concentration was used as polyanion. The slides were prepared by the Radio Corporation of America cleaning process. ${ }^{11}$ Several samples were fabricated with PAH and PCBS solutions of different $p \mathrm{H}$ levels. The $p \mathrm{H}$ of the $\mathrm{PAH}$ solutions ranged from 6 to 9 and that of the PCBS solutions ranged from 6 to 8 , respec-

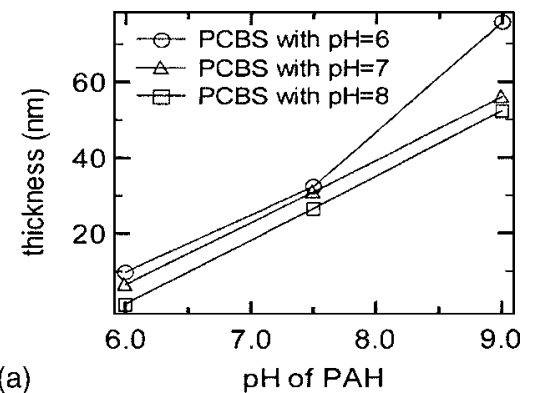

(a)

(a)

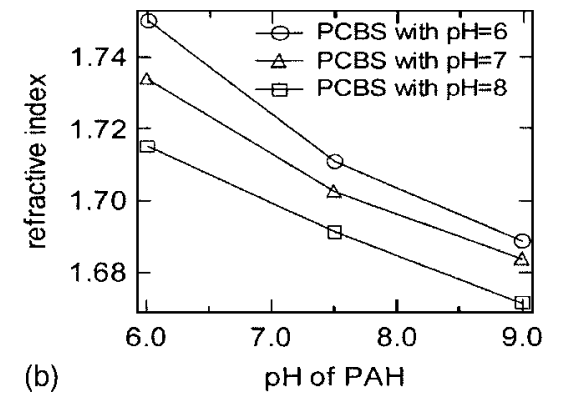

FIG. 2. Thickness and refractive index of 20 bilayer PAH/PCBS ISAM films on glass slides measured by ellipsometry. (a) Thickness vs $p \mathrm{H}$ value of PAH. (b) Refractive index vs $p \mathrm{H}$ value of PAH.

tively. All samples were prepared by depositing 20 bilayers of PAH/PCBS on the slides.

Figure 2 shows the values of total thickness $d_{\text {tot }}$ and refractive index $n$ as a function of $p \mathrm{H}$ of the polyanion/cation solutions. When the $p \mathrm{H}$ of the PCBS solution is fixed, $d_{\text {tot }}$ increases while $n$ decreases as the $p \mathrm{H}$ of the PAH of the PCBS solution increases. In contrast, when the $p \mathrm{H}$ of the PAH solution is fixed, both $d_{\text {tot }}$ and $n$ decrease as the $p \mathrm{H}$ of the PCBS solution increases. The reason for this is that both PAH and PCBS are weak electrolytes such that variations of $p \mathrm{H}$ will change the charge density of the adsorbing polymer. ${ }^{12,13}$ This behavior is understood as follows-both solutions are fully charged at neutral $p \mathrm{H}$. As the $p \mathrm{H}$ is lowered from neutral, the charge density of PCBS decreases since its acid groups become protonated. While, as the $p \mathrm{H}$ of the PAH solution is increased, its ammonium groups become deprotonated, and the charge density of PAH will decrease. As charge density decreases, less repulsion between neighboring charges allows more polymer chains to be adsorbed on the substrate with loops and tails to induce thicker layers. Furthermore, since PCBS has a higher refractive index than PAH, a higher percentage of PCBS results in a higher composite index of the ISAM film while a higher percentage of PAH results in lower composite index. Therefore, the thickness and refractive index of ISAM films can be fine tuned by adjusting the $p \mathrm{H}$ values of the polyelectrolyte solutions.

Note that the thickness of the films described in the previous section is of the order of several nm, and their "bulk" refractive indices are significantly higher than that of silica. Normally, this regime would not be very interesting for LPG tuning applications, since LPGs are most responsive to ambient index changes for bulk refractive index values slightly lower than silica. To test the influence of ISAM films on LPGs, different $p \mathrm{H}$ values for the PAH and PCBS solutions were utilized in fabricating films. As noted earlier, this film deposition process is agnostic to substrate topology or geometry, and thus obtaining cylindrically symmetric ISAM films on fibers requires no additional setup. The $p \mathrm{H}$ of the $\mathrm{PAH}$ 

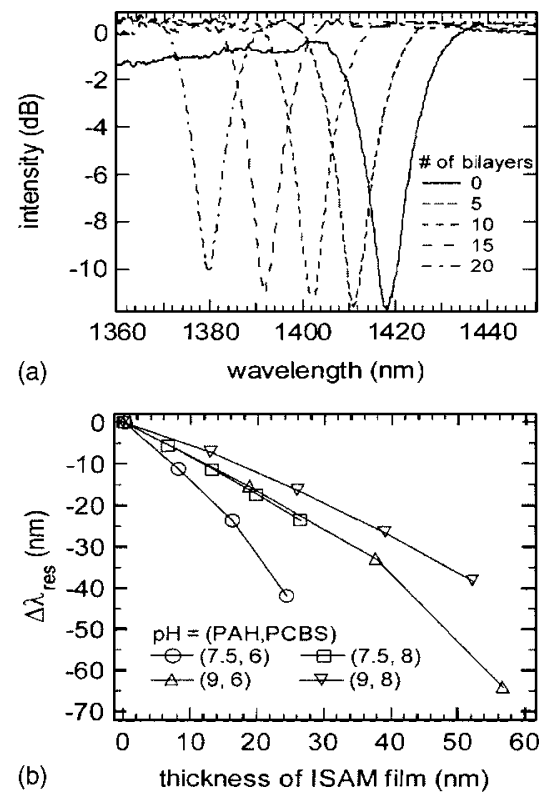

FIG. 3. Experimental results of ISAM film coatings on a LPG. (a) LPG transmission spectra with $0,5,10,15$, and 20 bilayers of PAH/PCBS ISAM film for PAH solution $p \mathrm{H}$ of 9 and PCBS solution $p \mathrm{H}$ of 8. (b) $\Delta \lambda_{\text {res }}$ of LPG as functions of thickness of ISAM film with different $p \mathrm{H}$ combinations of PAH and PCBS $[(7.5,6),(7.5,8),(9,6)(9,8)]$ which offer corresponding refractive index $n[1.7107,1.6912,1.6887,1.6715]$, respectively.

solutions for this experiment varied from 7.5 to 9 , while the $p \mathrm{H}$ of PCBS solutions ranged between 6 and 8. The LPGs were UV-induced on TrueWave ${ }^{\mathrm{TM}} \mathrm{RS}$ fibers with a grating period of $116 \mu \mathrm{m}$ and a length of $5 \mathrm{~cm}$. This yielded gratings that couple the fundamental mode to the $L P_{0,12}$ cladding mode at a resonant wavelength of $1420 \mathrm{~nm}$. The transmission spectra were measured by an optical spectrum analyzer after deposition of every five PAH/PCBS bilayers.

The experimental results of the ISAM-coated LPG are shown in Fig. 3. Figure 3(a) shows an example of the shift in the LPG resonance as the number of bilayers of the ISAM film (and, hence, its thickness) is increased. Note that the shift in the resonance is reminiscent of shifts attributed to increasingly higher ambient index values for an LPG in silica fiber, with the ambient index value remaining lower than that of silica. This is further illustrated in Fig. 3(b), which shows the change in resonant wavelength as a function of the film thickness for a variety of $p \mathrm{H}$ combinations of PAH and PCBS solutions. A record steep resonant wavelength shift of $1.6 \mathrm{~nm} / \mathrm{nm}$ is obtained for the case where the $p \mathrm{H}$ of PAH is 7.5 and that of PCBS is 6.

According to the LPG resonant condition equation

$$
\lambda_{\text {res }}=\left(n_{\text {core }}-n_{\text {clad }}\right) \Lambda \text {, }
$$

where $\lambda_{\text {res }}$ is the resonant wavelength, $n_{\text {core }}$ is the effective index of the fundamental core mode, $n_{\text {clad }}$ is the effective index of the coupled cladding mode, and $\Lambda$ is the grating period. Since it is the effective index of the coupled cladding mode that is significant, it is possible to observe shifts of $\lambda_{\text {res }}$ when thin films with index larger than that of the cladding are deposited on the cladding exterior. This is in contrast to the case in which the fiber is immersed in a bulk medium of higher index. In the latter case, there is diminished coupling which cannot be spectrally tuned. This is because the higher ambient index medium frustrates total internal reflection (TIR) at the glass-cladding surface, a condition necessary for the existence of weli-guided clading modes. In such cases, à substantially weak resonance is obtained. Moreover, changes in the index of the ambient would not cause spectral shifts. This is because a leaky mode is not guided due to TIR, but exists because of (weak) Fresnel reflections at the glassISAM boundary, and such reflections have no spectral phase dependence. The data of Fig. 3 show, for example, that $\lambda_{\text {res }}$ shifts due to changes in index as well as thickness of the ISAM films. The changes in ISAM deposition conditions (leading to changes in their indices) clearly produce spectral shifts of strong resonances for index values larger than that of the cladding. This may be understood by considering the mechanism of coupling in ISAM coated LPGs.

The evanescent tail of the cladding-mode field normally extends by $100-200 \mathrm{~nm}$ into the ambient medium. Since all the ISAM films we investigated are thinner than this value, the cladding mode interrogates both the ISAM as well as the surrounding air. The effective index $n_{\text {eff }}$ of a guided mode can be approximated by ${ }^{14}$

$$
n_{\mathrm{eff}}^{2} \sim \iint n^{2}(r) \cdot|E(r)|^{2} \cdot d A
$$

where $E(r)$ is the mode field distribution, $n(r)$ is the refractive index profile of the fiber, and $\iint d A$ signifies an integration across the cross-sectional area of the fiber. Equation (2) shows that the effective index of a mode is approximately proportional to the average refractive index of the region in which light exists, weighted by the local intensity profile. Since a typical cladding mode samples both the high index ISAM as well as the surrounding (low index) air, the average index of an ambient comprising 10-50-nm-thick ISAM films and air is that of an equivalent bulk medium with index lower than silica. Moreover, this average index value will depend on the film thickness in addition to the index of the film, with thicker films leading to a larger ISAM contribution to the average index, as is evident from Eq. (2).

In summary, we have demonstrated deposition of nmthick ISAM films on LPGs, which yields properties useful both as LPG tuning mechanisms as well as platforms for sensing devices. Fine control of the refractive index and the thickness of the ISAM film was achieved by altering the relative fraction of the anionic and cationic materials deposited layer-by-layer. This highly controllable deposition technique facilitates fine-tuning grating properties. ISAM films with subwavelength thicknesses cause dramatic resonant wavelength shifts of LPGs, which enables their use as sensors of target compounds.

${ }^{1}$ A. M. Vengsarkar, P. J. Lemaire, G. Jacobovitz-Veselka, V. Bhatia, and J. B. Judkins, Proc. IOOC'95, PD1-2, 1995.

${ }^{2}$ S. Ramachandran, S. Ghalmi, Z. Wang, and M. Yan, Opt. Lett. 27, 1678 (2002).

${ }^{3}$ S. Ramachandran, Z. Wang, and M. Yan, Opt. Lett. 27, 698 (2002).

${ }^{4}$ H. J. Patrick, A. D. Kersey, and F. Bucholtz, J. Lightwave Technol. 16, 1606 (1998)

${ }^{5}$ B. Acharya, T. Krupenkin, S. Ramachandran, Z. Wang, C. Huang, and J. Rogers, Appl. Phys. Lett. 83, 4912 (2003).

${ }^{6}$ Z. Wang and S. Ramachandran, Opt. Lett. 28, 2458 (2003).

${ }^{7}$ K. Tiefenthaler and W. Lukosz, Opt. Lett. 10, 137 (1984).

${ }^{8}$ N. Rees, S. James, R. Tatam, and G. Ashwell, Opt. Lett. 27, 686 (2002).

${ }^{9}$ G. Decher, Science 277, 1232 (1997).

${ }^{10}$ J. R. Heflin, C. Figura, D. Marciu, Y. Liu, and R. Claus, Appl. Phys. Lett. 74, 495 (1999).

${ }^{11}$ W. Kern and D. A. Poutinen, RCA Rev. 31, 187 (1970).

${ }^{12}$ S. Shiratori and M. Rubner, Macromolecules 33, 4213 (2000).

${ }^{13}$ C. Figura, P. J. Neyman, D. Marciu, C. Brands, M. A. Murray, R. M. Davis, M. B. Miller, and J. R. Heflin, Proc. SPIE 3939, 214 (2000).

${ }^{i 4}$ S. Ramachandran, J. Lightwave Technol. 28 (to be published). 